\title{
Genetic Programming Based Automatic Gait Generation for Quadruped Robots
}

\author{
Kisung Seo \\ Electronic Engineering \\ Seokyeong University \\ Seoul, Korea \\ 82-2-940-7162 \\ ksseo@skuniv.ac.kr
}

\author{
Soohwan Hyun \\ Electronic Engineering \\ Seokyeong University \\ Seoul, Korea \\ 82-2-940-7162 \\ xjavalov@shhyun.com
}

\begin{abstract}
This paper introduces a new approach to develop a fast gait for quadruped robot using genetic programming (GP). Several recent approaches have focused on the genetic algorithm (GA) to generate a gait automatically and shown significant improvements over previous results. Most of current GA based approaches use pre-selected parameters, but it is difficult to select the appropriate parameters for the optimization of gait. To overcome these problems of GA based approach, we proposed an efficient approach which optimizes joint angle trajectories using genetic programming. Our GP based method has obtained much better results than GA based approaches for experiments of Sony AIBO ers-7 in Webots environment. The elite archive mechanism(EAM) was introduced to prevent premature convergence problems in GP and has shown improvements.
\end{abstract}

\section{Categories and Subject Descriptors}

I.2.9 [Artificial Intelligence]: Robotics

\section{General Terms}

Algorithms, Design, Performance

\section{Keywords}

Genetic Programming, Quadruped Robot, Automatic Gait Generation, Elite Archive Mechanism

\section{INTRODUCTION}

The mobility of walking robot distinguishes from wheeled robot in that the walking robot can traverse in uneven and unstructured environments[1]. Planning gaits for quadruped robots, such as Sony Aibo, is a challenging task that requires optimizing a locus of robot's paw, an initial position and a number of steps in a highly irregular and multidimensional space.

Several recent works[1-4] have focused on genetic algorithm or evolutionary algorithm to automate gaits from optimizing initial position, a number of movement points per cycle, and the locus of paw(e.g., rectangular, elliptic, trapezoid, and three dimensional shape). However, the type and number of parameters related to the locus of paw and initial position are not standardized or fixed, so that the optimization results of a gait can be affected by

Copyright is held by the author/owner(s).

GECCO'08, July 12-16, 2008, Atlanta, Georgia, USA.

ACM 978-1-60558-130-9/08/07. selections of those parameters. Moreover, the number of parameters used in GA approaches are very large, ten to twenty at least. Additionally, an inverse kinematics calculation is necessary to convert the locus of paw into joint angles as extra efforts.

Therefore, in order to fundamentally overcome these problems of GA based approach, we propose an efficient approach with genetic programming to optimize joint angle trajectories instead of locus of paw. The proposed method optimizes only 4-6 joint trajectories rather than numerous parameters as in the GA approaches. Especially, GP based search is an effective way to generate each joint trajectories in an open-ended manner. Additionally, we introduce the modified elite archive mechanism(EAM), which can prevent premature convergence problems in GP.

\section{GP BASED GAIT CONTROL IN GAIT CONTROL IN JOINT SPACE}

The concept of gait generation in joint space using GP is shown in Figure 1. Without a need of conversion from Cartesian space, gait is determined by a series of joint positions(or angles) directly in terms of a numeric expression which represents joint trajectory by time. GP generates and evolves joint trajectories which are represented by each tree as similar to a symbolic regression.

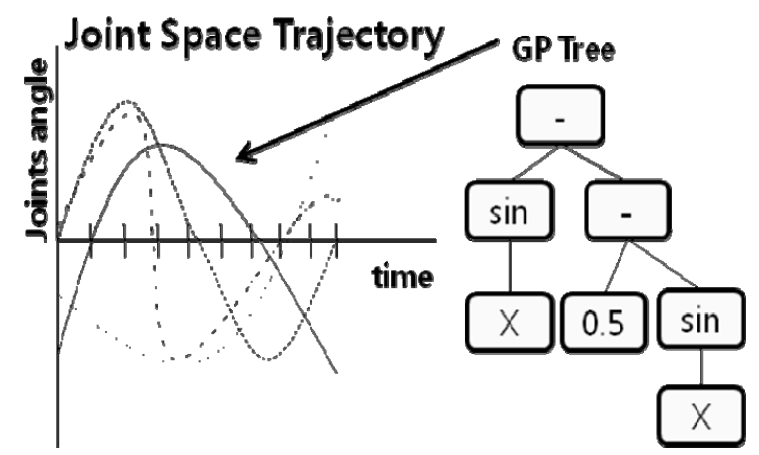

Figure 1. Representation of trajectory of joints and GP tree

The Sony Aibo has three joints - shoulder, flap, and knee - in each of its legs. Among three kinds of joints, only shoulder and knee are concentrated on a gait for a forward walk. Therefore, four joints trajectories, each with two joints for front and back, are enough to construct a gait for quadruped robot. In order to 
represent four joints trajectories for a gait using GP, multiple trees are used to compose an individual.

\section{GENETIC PROGRAMMING WITH ELITE ARCHIVE MECHANISM}

An elite archive mechanism is used to improve search capability of GP, while preventing a premature convergence in GP. This technique preserves elite individuals in the early stage and flow them into in later stage. Genetic materials of the elite individuals in early stage are used to refresh an evolutionary convergent state and to make a role to preserve diversity as long as possible.

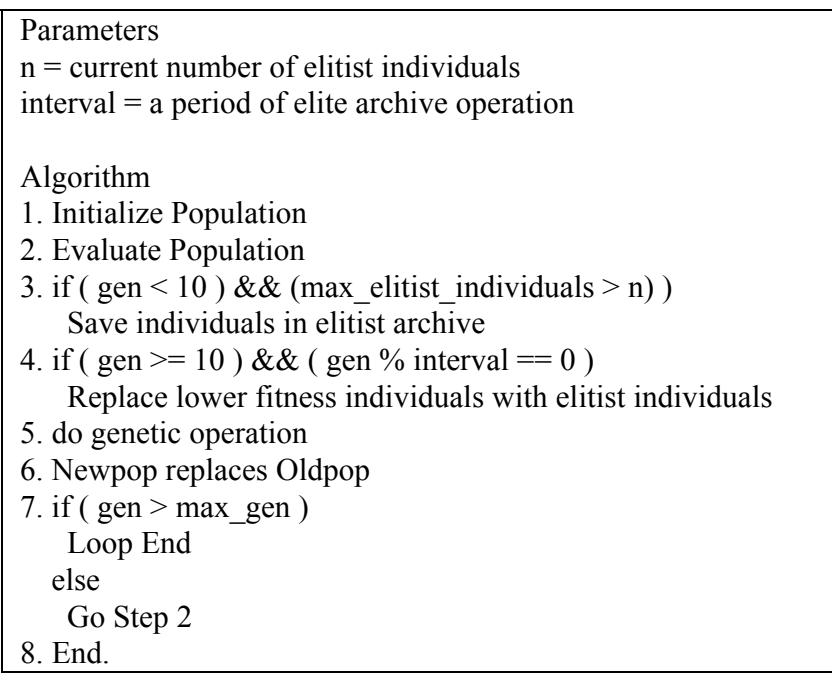

\section{EXPERIMENTS}

A simulated model of Sony Aibo ERS-7 in Webots[5] is used. The fitness function of gait generation is defined to obtain fast walking velocity within a small sideway diversion. A trot gait is selected as was in other approaches.

The tabular results of average velocities for generated gaits are provided in Table 1. Every GP run is repeated 10 times for each case of the experiments. The velocities are measured by iterating 8 cycles of gaits, which approximately covers a moving distance of $1.8 \mathrm{~m}-2 \mathrm{~m}$.

Table 1. Velocities by variation of pop sizes for multi-pops and $\operatorname{EAM}(\mathbf{c m} / \mathbf{s})$

\begin{tabular}{|c|c|c|}
\hline Pop size & Multi-pops & Multi-pops with EAM \\
\hline $20 * 5$ & 50.63 & 54.15 \\
\hline $50 * 5$ & 52.74 & 58.35 \\
\hline $100 * 5$ & 50.65 & 61.02 \\
\hline
\end{tabular}

The results of ordinary multi-pops with GP have shown around 50 $\mathrm{cm} / \mathrm{s}$ which are slightly better than the recent GA's approach[2]. The results by elite archive mechanism(EAM) GP are much better than those of multi-pops approach. It seems to be more robust for a premature convergence problem.

To illustrate the obtained gaits in detail, joint trajectories and gait motions for a typical evolved solution is displayed in Figure 2 and 3. FJ1 and FJ2 represent a joint of front shoulder and front knee, BJ1 and BJ2 means a joint of back shoulder and back knee.

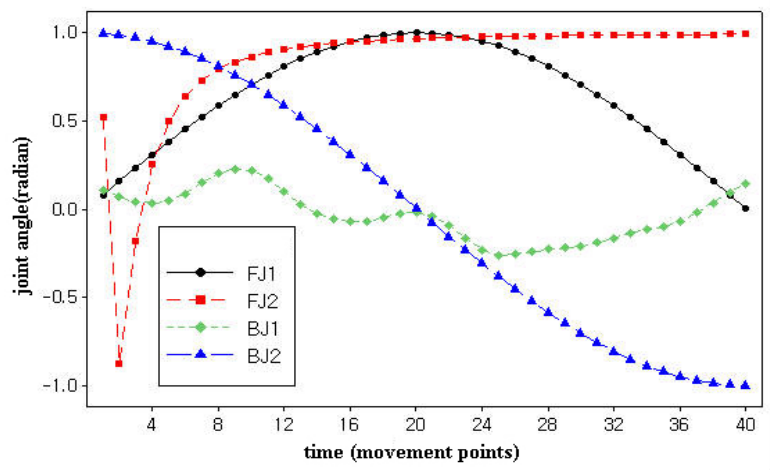

Figure 2. Joint trajectory graph $(1$ point $=8 \mathrm{~ms})$

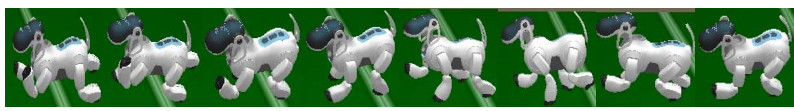

Figure 3. Gait motions

\section{ACKNOWLEDGMENTS}

This work was supported by the Korea Research Foundation Grant funded by the Korea government (MOFHRD) Basic Research Promotion Fund) (KRF-2007-314-D00176)

\section{REFERENCES}

[1] Hornby, G. S., Takamura, S., Yamamoto, T., Fujita, M. Autonomous evolution of dynamic gaits with two quadruped robots. IEEE Trans. Robotics, Vol. 21, No. 3, 2005, 402-410.

[2] Dong, H., Zhao, M., Zhang, J., Shi, Z., Zhang, N. Gait planning of quadruped robot based on third-order spline interpolation. In Proceedings of the 2006 IEEE/RSJ International Conference on Intelligent Robots and Systems(IROS'06), Beijing China October 9 - 15, 2006.

[3] Chen, W. Odometry Calibration and Gait Optimisation. Technical Report, The University of New South Wales, School of Computer Science and Engineering, 2005.

[4] Mericli, T., Akin, H. L., Mericli, C., Kaplan, K., Celik, B. The Cerberus'05 Team Report.

[5] Hohl, L., Tellez, R., Michel, O., Ijspeert, A. J. Aibo and Webots: Simulation, wireless remote control and controller transfer. Robotics and Autonomous Systems, 54, 2006, 472485 . 